\title{
Actitudes de los docentes frente a la inclusión de estudiantes con necesidades educativas especiales
}

\section{Yineida González-Rojas}

orcid.org/oooo-0003-1642-7992

Universidad de la Amazonía, Colombia

yin.gonzalez@udla.edu.co

\section{Dairo Alberto Triana-Fierro}

orcid.org/00oo-0002-5490-3673

Universidad de la Amazonía, Colombia da.triana@udla.edu.co

\section{Resumen}

Este artículo tiene como objetivo principal la revisión de publicaciones especializadas que abordan los factores influyentes en las actitudes de los docentes hacia la inclusión de estudiantes con necesidades educativas especiales, en lo que va corrido del siglo XXI. Inicialmente, se delimita el tema respecto a la definición de educación inclusiva, necesidades educativas especiales y alumnos con dichas necesidades, desde la normatividad colombiana y la posición de organizaciones internacionales. Seguidamente, se expone el término actitud y sus tres componentes principales: cognoscitivo, afectivo y conductual, para luego profundizar en los siete factores que según los expertos pueden influenciar las actitudes de los profesores hacia los alumnos con necesidades educativas especiales: responsabilidad, rendimiento, formación y recursos, clima de aula, relación social, desarrollo emocional y creencias. Se concluye reflexionando sobre las potencialidades que tienen docentes, directivos docentes e instituciones educativas para que la inclusión brinde los resultados favorables que de ella se esperan en la formación integral de los estudiantes.

\section{Palabras clave}

Actitud del docente, alumno atípico, educación especial, integración educativa, necesidades educativas (Fuente: Tesauro de la UNESCO). 


\title{
Attitudes of Teachers on Including Students with Special Educational Needs
}

\begin{abstract}
The main objective of the article is to review specialized publications that deal with the factors that influence the attitudes of teachers, so far during the $21^{\text {st }}$ century, when it comes to including students with special educational needs. At first, the subject is defined or limited by the definition of inclusive education, special educational needs and students with those needs, viewed from the standpoint of Colombian regulations and the position of international organizations. The article then goes on to look at the term attitude and its three main components: cognitive, affective and behavioral, before delving into the seven factors that experts say can influence the attitudes of teachers towards students with special educational needs: namely, responsibility, performance, training and resources, the climate in the classroom, social relationship, emotional development and beliefs. The article concludes with reflections on the potential of teachers, teaching administrators and educational institutions to ensure that inclusion provides the favorable results expected of it in terms of comprehensive education for students.
\end{abstract}

\section{Keywords}

Teacher's attitude, atypical student, special education, educational integration, educational needs (Source: UNESCO Thesaurus). 


\section{Atitudes dos docentes frente à inclusão de estudantes com necessidades educativas especiais}

\section{Resumo}

O artigo tem como objetivo principal a revisão de publicações especializadas que abordam os fatores influentes nas atitudes dos docentes sobre a inclusão de estudantes com necessidades educativas especiais, no transcurso do século XXI. Inicialmente, o tema é delimitado ao que diz respeito à definição de educação inclusiva, necessidades educativas especiais e alunos com tais necessidades, a partir da normatividade colombiana e da posição de organizações internacionais. Em seguida, o termo "atitude" e seus três componentes principais são expostos: cognoscitivo, afetivo e comportamental, para, logo, centrar-se nos sete fatores que, segundo os especialistas, podem influenciar as atitudes dos professores com os alunos que possuem necessidades educativas especiais: responsabilidade, rendimento, formação e recursos, clima de aula, relação social, desenvolvimento emocional e crenças. O artigo é concluído com uma reflexão sobre as potencialidades que os docentes, diretivos docentes e instituições educativas têm para que a inclusão ofereça os resultados favoráveis que são esperados dela na formação integral dos estudantes.

\section{Palavras-chave}

Atitude do docente, aluno atípico, educação especial, integração educativa, necessidades educativas (Fonte: Thesaurus da Unesco). 


\section{Introducción}

El término educación inclusiva, o inclusión educativa, hace referencia al derecho que toda persona tiene a una educación de calidad e implica el hecho de que ser diferente es algo legítimo. Para educar en la inclusión y responder a las Necesidades Educativas Especiales (NEE) de los estudiantes, es necesario identificarlas y abordarlas realizando los cambios pertinentes en los contenidos curriculares, instalaciones locativas, materiales de aprendizaje y preparación de los docentes. Debe entonces considerarse la educación inclusiva como la posibilidad de ofrecer un entorno educativo que se caracterice por un ambiente profesional sensible a las necesidades y demandas específicas de todos los alumnos que les permita el aprendizaje significativo de los contenidos curriculares, pero también desarrollar sus habilidades sociales y emocionales como seres humanos capaces de sentir y mostrar sus sentimientos, aún y cuando entre ellos exista algún tipo de NEE.

La Constitución Política de 1991 en sus artículos 13, 47 y 98 establece los lineamientos de la Política de Educación Inclusiva en Colombia, reglamentada posteriormente por la Ley General de Educación 115 de 1994 y el Decreto 2082 de 1996. En términos generales, la normatividad promulga el derecho a la igualdad y a la no discriminación y la obligación del Estado de proveer a las instituciones y educadores de los recursos y capacitación necesarios para atender a los alumnos con NEE. Así mismo, en su artículo 5, la Constitución Política de 1991 reconoce que las personas con NEE tienen derecho a acceder a una educación de calidad a lo largo de toda su vida, que promueva su desarrollo integral, su independencia y su participación, en condiciones de igualdad, en los ámbitos público y privado.

Se define como estudiantes con NEE a aquellos con capacidades excepcionales, o con alguna discapacidad de orden sensorial, neurológico, cognitivo, comunicativo, psicológico o físico-motriz, que puede expresarse en diferentes etapas del aprendizaje
(Decreto 366 de 2009, art. 2). La Ley 115 de 1994, en su título III, capítulo I, artículos 46 al 48, regula la atención educativa de las personas con limitaciones de orden físico, sensorial, psíquico, cognoscitivo o emocional como parte del servicio público educativo. El Decreto 2082 de 1996 reglamentario de la Ley 115, en su artículo 12 establece que los departamentos, distritos y municipios organizarán en su respectiva jurisdicción un plan de cubrimiento gradual para la adecuada atención educativa de las personas con limitaciones o con capacidades o talentos excepcionales, el cual hará parte del plan de desarrollo educativo territorial.

El establecimiento y puesta en marcha de esta Política de Educación Inclusiva ha generado cambios importantes dentro de las instituciones educativas y los profesores son protagonistas de primer orden en su desarrollo e implementación. Por ello, el interés de este artículo se centra en las actitudes que tienen hacia la inclusión de estudiantes con NEE en sus aulas y en los factores que pueden influir en dichas actitudes.

\section{Metodología}

Se realizó una revisión bibliográfica exhaustiva sobre el tema en diversos motores de búsqueda, de los cuales Google Académico, como buscador especializado en búsqueda de contenido y literatura científico-académica, brindó información relevante de trabajos publicados en bases de datos tales como Scielo, Redalyc y Dialnet. Posteriormente, se realizó la búsqueda de información en bases de datos especializadas, como Scopus, Web of Science, así como en diversos repositorios universitarios. Las palabras utilizadas para esta revisión bibliográfica pertenecen a las categorías: actitud del docente, educación especial, necesidades educativas, y a las metacategorías: integración educativa y alumno atípico. En esta búsqueda, los registros obtenidos oscilaron entre 147 y 58, tras la combinación de las diferentes palabras. 
Los criterios para la selección de la información fueron: pertinencia, relación con el tema objeto de estudio y que estuvieran enmarcados temporalmente en el nuevo milenio. Por tanto, los documentos seleccionados pertenecen a revistas especializadas y trabajos de grado referentes al área en estudio. Seguidamente, se seleccionaron los documentos que informaran sobre aspectos formales e incluyeran una revisión bibliográfica del tema de estudio. Para ello se tuvieron en cuenta: los textos, como apoderados de la experiencia, con análisis comparativos, análisis de palabras y códigos. También se tuvieron en cuenta como objeto de análisis: ejecuciones, conversaciones, estructuras gramaticales y narrativas.

\section{Resultados}

El tema cobra importancia, si se considera que las primeras experiencias académicas que se tienen en la infancia son fundamentales en la valoración que del hecho de aprender hace cada ser humano. Por ello, es importante que la llegada a las aulas, las clases y los procesos de aprendizaje se conviertan en un hecho placentero, tanto para los alumnos regulares como para aquellos con NEE, de modo que genere inquietud por el descubrimiento de los nuevos saberes y motivación para aprender más.

La inclusión significa atender con calidad y equidad las necesidades comunes y específicas que presentan los estudiantes (MEN, 2007, p. 1). Por su parte, la Organización de las Naciones Unidas para la Educación, la Ciencia y la Cultura (Unesco, 2009, citado en Latorre, 2013, p. 96) define la educación inclusiva como un proceso que comporta la transformación de las escuelas y de otros centros de aprendizaje para atender a todos, esto es: a los niños y niñas, a los alumnos que pertenecen a grupos étnicos y lingüísticos minoritarios o a poblaciones rurales, a los afectados por el VIH y el SIDA o con discapacidad y dificultades de aprendizaje. Además, se señala que la educación ha de brindar también oportunidades de aprendizaje a todos los jóvenes y adultos. Tiene por objetivo acabar con la exclusión que es conse- cuencia de actitudes negativas y de una falta de atención a la diversidad en materia de raza, situación económica, clase social, origen étnico, idioma, religión, sexo, orientación sexual y aptitudes.

El concepto de educación inclusiva pone en tela de juicio gran parte del modo de organizar y disponer la enseñanza de las escuelas tradicionales. Aunque las instituciones deben tener objetivos generales o comunes en cuanto a lo que es adecuado y deseable que los educandos logren aprender, las competencias relacionadas con las distintas materias escolares se deben considerar en el contexto de las oportunidades y necesidades de cada educando (Unesco, 2009, en Latorre, 2013, p. 20). El desarrollo de una educación inclusiva implica cambios en el ámbito del sistema y de las políticas educativas, en el funcionamiento de las instituciones académicas, en las actitudes y prácticas de los docentes y en los niveles de relación de los distintos actores que permitan enfrentar y eliminar las barreras para el aprendizaje y la participación, las cuales se concentran en las siguientes realidades:

- La formación docente, que no siempre prepara al educador para abordar las necesidades individuales de sus estudiantes.

- El diseño curricular y los sistemas de promoción y evaluación, que tienden a implementar modelos estandarizados no inclusivos.

- La ausencia de recursos técnico-pedagógicos complementarios que refuercen la capacidad de la institución para dar respuesta a la diversidad.

- Barreras culturales y actitudinales que tienen que ver con comportamientos discriminatorios por parte de otros niños, de sus familias o de integrantes del equipo docente (Latorre, 2013, p. 98).

Una de las premisas más importantes para la consecución de la inclusión educativa es el cambio en la forma de enseñanza y conlleva la capacitación 
de los profesores para que aprendan a trabajar con personas que se salen de lo preestablecido. Es importante el rol del docente como agente educativo central de este proceso. Por tanto, es necesario que este desarrolle actitudes positivas, mediante las cuales estos estudiantes se sientan miembros de una familia y también de una comunidad. Esto lo logrará aumentando su autoestima, haciendo un esfuerzo por incrementar su autoconfianza a través de las interacciones positivas entre los miembros de clase (incluido el profesor), creando situaciones en las que los alumnos puedan colaborar, experimentar y aprender juntos y, además, incluyendo destrezas pedagógicas adecuadas y tiempo para la reflexión profesional, así creará sus propias destrezas en el área (Universidad de Panamá, 2016).

Lo expuesto hasta aquí ratifica que el docente juega un papel muy importante en la educación inclusiva y, por tanto, su actitud es un eslabón primordial para el éxito. Al respecto, durante los últimos cuarenta años se han llevado a cabo numerosas investigaciones acerca de las características de edad, clase social, experiencia, salario, entrenamiento recibido y actitud que deben tener los maestros eficientes e incluyentes. Desde estos planteamientos se aborda el concepto de actitud, primero de forma global, para luego profundizar en la actitud del profesorado y los factores que pueden influir en ella.

\section{Aproximación al término actitud}

Stahlberg y Frey (1991, citados en Castillo, 2007) definen la actitud como un factor que determina diferentes situaciones y lleva al individuo, por ejemplo, a tomar decisiones o reaccionar a cierta situación. Lo conduce a actuar de una forma concreta y específica, y normalmente esta forma es constante, es decir, permanece. Baron y Byrne (2002) ven la actitud como reacción afectiva positiva o negativa hacia un objeto o proposición abstracta o concreta y señalan que las actitudes son aprendidas, tienden a permanecer bastante estables con el tiempo, están dirigidas siempre hacia un objeto o idea particular y poseen tres componentes:
- $\quad$ Cognoscitivo: está formado por las percepciones y creencias respecto de un objeto, así como por la información que se tiene del mismo. Los objetos no conocidos o sobre los que no se posee información no pueden generar actitudes. La representación cognoscitiva puede ser vaga o errónea. En el primer caso, el afecto relacionado con el objeto tenderá a ser poco intenso, y cuando es errónea no afectará para nada la intensidad del afecto. El componente cognoscitivo de la actitud comprende las percepciones, las opiniones y las creencias de las personas. Se refiere al proceso del pensamiento, con especial énfasis en la racionalidad y en la lógica. Un elemento importante de la cognición es el de las creencias evaluativas que mantiene la persona. Las creencias evaluativas se manifiestan como impresiones favorables o desfavorables que alguien mantiene respecto de un objeto o una persona.

- Afectivo: es el sentimiento en favor o en contra de un objeto social. Es el componente más característico de las actitudes. Aquí radica la diferencia principal con las creencias y las opiniones, que se caracterizan por su componente cognoscitivo. El componente emocional o sentimental de una actitud se aprende de los padres, de los maestros y de los camaradas.

- Conductual: es la tendencia a reaccionar ante los objetos de una determinada manera. Es el componente activo de la actitud. El componente actitudinal de la conducta se refiere a la tendencia de la persona a actuar frente a algo o ante alguien de una manera determinada. La medida de estas acciones puede ser útil para examinar los componentes de la conducta en las actitudes. Las actitudes son determinantes de la conducta, ya que están ligadas a la percepción, a la personalidad y a la motivación. Una actitud es un sentimiento o estado mental positivo o negativo de disposición, conseguido y organizado a través de la experiencia, 
que ejerce una influencia específica en la respuesta de la persona a los demás, a los objetos $y$ a las situaciones.

Las actitudes de los profesores hacia los alumnos con NEE pueden verse influenciadas por varios factores que Mula et al. (2002) estudiaron detenidamente, hasta llegar a establecer como principales los siguientes:

- Responsabilidad: se refiere a la opinión de los docentes en cuanto a quiénes son los encargados de formar a los estudiantes con NEE y de elaborar, poner en práctica y realizar seguimiento a las adaptaciones curriculares.

- Rendimiento: se trata de la posición de los edu cadores frente al rendimiento académico de los alumnos con NEE, al recibir formación en una misma aula con otros estudiantes.

- Formación y recursos: se define como las consideraciones de los profesores respecto a su propia formación y conocimientos, así como los recursos con que cuentan para atender a los alumnos con NEE.

- Clima de aula: es la opinión de los docentes en cuanto a la disciplina y el orden en el aula de clase compartida por estudiantes con NEE y alumnos regulares.

- $\quad$ Relación social: se refiere a los beneficios percibidos por los educadores respecto a la socialización de los alumnos con NEE gracias a la integración en el aula.

- Desarrollo emocional: es la posición de los profesores frente a la integración en el aula como elemento que contribuye o no en el desarrollo emocional de los estudiantes con NEE, entendido como el proceso de formación de su identidad, autoestima y confianza en sí mismos.

- Creencias: opinión de los educadores frente a la integración en el aula como una práctica educativa deseable.
Teniendo en cuenta estos factores, se incluyen a continuación aportes destacados de la revisión bibliográfica, que los analizan y brindan sugerencias para que la integración al aula regular de los estudiantes con NEE logre darse favorablemente.

\section{Factores influyentes en las actitudes del profesorado hacia los estudiantes con NEE}

RESPONSABILIDAD: la formación de los alumnos con NEE es responsabilidad de los docentes; sin embargo, dicha formación es también corresponsabilidad de otros actores educativos, como los directivos docentes y psicopedagogos. Al respecto, Duk y Loren (2013, p. 192) destacan la importancia, por un lado, de la adaptación curricular como respuesta a la diversidad, materializada en un continuo de ajustes que deben realizar las instituciones educativas a través de sus docentes a los planes y programas de estudio, en particular para aquellos estudiantes que se encuentren en riesgo de fracaso, y por otro, de la gestión de sistemas y procedimientos de evaluación y promoción que valoren los esfuerzos y logros individuales, de modo que la gestión se centre en el alumno/a y su aprendizaje.

Las autoras citan a Hineni (2008), quien describe las actividades que el conjunto de integrantes de la institución educativa debe llevar a cabo para el desarrollo de la adaptación curricular en beneficio de los estudiantes con NEE, así: programación curricular que incorpore la diversidad de necesidades educativas de los estudiantes de forma que los aprendizajes sean pertinentes para todos; criterios y procedimientos establecidos por el conjunto de los docentes para adaptar y diversificar el currículo en función de las características y necesidades del alumnado; planificaciones de aula y su revisión periódica en forma colaborativa; docentes con los recursos de tiempo y apoyo para su elaboración; un sistema para identificar y evaluar a los estudiantes que enfrentan dificultades en su proceso educativo, orientado a proporcionarles los recursos y ayudas que requieran; revisión y evaluación de las prácticas 
educativas por parte de los docentes en colaboración con los profesionales de apoyo, identificando las barreras que dichas prácticas pueden suponer para los procesos de aprendizaje, buscando soluciones y alternativas que las mejoren, entre las que se cuentan los planes de refuerzo, los planes de nivelación y los planes educativos personalizados.

En Colombia, la adaptación curricular para atender a los estudiantes con NEE parte de un currículo flexible, definido por el Ministerio de Educación Nacional (2015) como aquel que conserva los objetivos generales de la enseñanza, pero otorga diferentes oportunidades para alcanzarlos con base en la diversidad, dando a todos los estudiantes la misma posibilidad de aprender. De este modo, la flexibilización curricular se da en tres niveles: inicialmente, a nivel macro, con lineamientos específicos que deben formularse en el Proyecto Educativo Institucional (PEI); seguidamente, con su puesta en marcha en las aulas de clase, mediante el diseño de estrategias para enseñar y evaluar; y finalmente, en adaptaciones curriculares específicas e individualizadas con los alumnos que así lo requieren. Plantea el Ministerio que los ajustes y adaptaciones curriculares en Colombia pueden ser organizativos, didácticos y de área, y aplicables a las actividades, asignaturas, contenidos, horarios, intensidad de la jornada escolar, logros, tiempo para alcanzarlos y tipo de evaluación.

Sin embargo, existen profesores que aún creen que la responsabilidad de la formación de los estudiantes con NEE recae en el maestro de apoyo o en especialistas, aferrados como están a su rol tradicional, el cual está vinculado a ser un "transmisor de información" a un sujeto pasivo que debe asimilar lo entregado. Ello indica que no están preparados para el trabajo con alumnos con NEE, porque se han acostumbrado a trabajar con niños "normales", lo que permite inferir que estos docentes se estarían marginando del proceso de inclusión (Vega, 2009, p.190). Así lo evidencia el estudio realizado por Medina (2015) con profesores de dos instituciones públicas del departamento de Boyacá, quienes, según la autora, parecieran desconocer o ignorar en su práctica pedagógica la importancia de las adaptaciones curriculares en alguno o todos los elementos constitutivos del currículo: propósitos, competencias, métodos y evaluación, para responder a las condiciones particulares de estos estudiantes, a fin de promover y desarrollar su potencial de aprendizaje.

Rendimiento: Gavia (2013, p. 245) realizó un estudio en el cual llega a concluir que las creencias de los profesores respecto al rendimiento académico de los estudiantes con NEE están basadas en las características de los niños, en los sentimientos que provocan en ellos y en su capacidad para aprender. Estas creencias están circunscritas o fundamentadas en su experiencia laboral, más que en referencias actualizadas de las NEE. Kaplan (2004, p. 85) sostiene que las creencias de los profesores aluden a comprensiones, supuestos, imágenes o proposiciones sentidas como verdaderas, a partir de las cuales orientan sus acciones, apoyan sus juicios y toman decisiones, tipificando no solo lo que es, sino también lo que debería ser. Así mismo, las representan como un repertorio de supuestos y respuestas rutinarias a los problemas profesionales, sin base teórica que las sustente, los cuales determinan su comportamiento.

Las creencias docentes constituyen verdaderas teorías implícitas sobre el aprendizaje y la enseñanza y están profundamente enraizadas, no solo en la cultura escolar dominante y en las actividades de enseñanza cotidianas, en la organización del aula, en la evaluación, etc., sino también en la propia estructura cognitiva de profesores y alumnos. Encarnan así los referentes a los que acuden los profesores de manera consciente o inconsciente para organizar y tomar las consiguientes decisiones que guiarán las elecciones de las estrategias docentes y sus respuestas a las diferentes preocupaciones y prioridades (Pozo, 2000, p. 6, citado en Prieto, 2008, p. 129).

Los profesores no construyen esas creencias de manera intencionada y consciente; por el contrario, 
las han internalizado en sus estructuras cognitivas y emocionales de manera inconsciente a partir de una interpretación del significado otorgado a los procesos vividos en su cultura específica. Su origen puede fluctuar entre la idealización de experiencias pasadas, hasta creer simplemente que algo es correcto en una situación especial. Ello no implica necesariamente un error, sino el resultado de una realidad empírica: las creencias no pueden ni pretenden explicar la realidad, sino solo interpretarla subjetivamente acorde con el contexto de ocurrencia y la urgencia por solucionar algún problema surgido del proceso de enseñanza. De hecho, nadie puede negar que los acontecimientos en las aulas se suceden con tal celeridad que impiden a muchos profesores buscar certezas, ya que deben responder y resolver problemas prácticos de manera rápida y eficaz (Prieto, 2008, p. 130).

Las concepciones de los docentes sobre el rendimiento académico de sus estudiantes serían fruto de su saber pedagógico informal y estarían entonces ligadas a su experiencia en la interacción con los estudiantes y configuran la enseñanza, en la medida en que se desarrollan en escenarios socioculturales donde los docentes constituyen subjetividades (Casas y García, 2016, p. 136). López (2014, p. 103) indica que, ante las NEE, la pieza clave es el aire de positividad que debe existir en los profesores, y es necesario que cuestionen sus creencias, prejuicios y la zona de confort en la que desean mantenerse, puesto que la diferencia es la llave que abre las puertas a un universo de aprendizaje y de mejora y crecimiento personal. La falacia de la homogeneidad queda en entredicho cuando se aprende de aquellos que parecieran estar en desventaja, cuando la actitud de apertura permanece, más allá de las creencias, de los prejuicios y de los mitos, que deben ser desterrados del pensamiento de los profesores. El arraigo de las creencias de los profesores respecto de sus alumnos puede convertirse en un obstáculo a la hora de emprender acciones conducentes al beneficio de los estudiantes con NEE. Las creencias son el alimento de la resistencia al cambio, dado que este implica modificaciones tanto en la forma de pensar como en la de proceder. No es fácil moverse de lo que ya se conoce y se tiene como cierto, hacia lo desconocido, y resulta difícil para los docentes romper con sus rutinas e implementar nuevas prácticas.

Por lo anterior, en el acto de enseñar el docente asume un papel protagónico, pues en sus prácticas pedagógicas es el responsable de que los alumnos regulares y con NEE alcancen el desarrollo de competencias cognitivas y sociales. Y como su labor no se reduce a la ejecución de los contenidos y currículos, debe dejar de ser un personaje pasivo y, a partir de la reflexión, constituirse en un sujeto transformador, capaz de autoevaluarse, transformar el modelo tradicional y desarrollar en los estudiantes las habilidades que les permitan alcanzar el aprendizaje significativo.

FormaCión Y RECURSOS: las actitudes de los profesores frente a los alumnos con NEE también pueden verse influenciadas por sus percepciones en cuanto a los recursos y características de la institución, así como por su formación e iniciativa para trabajar con ellos. La formación de los docentes ha ido evolucionando conforme se presentan cambios en los sistemas educativos, cambios que cada día exigen nuevos perfiles del educador en los que la actividad de enseñar pase de lo teórico y lo técnico a un proceso reflexivo que permita imaginar y concretar espacios de instrucción realmente significativos, que además son, hoy por hoy, espacios incluyentes en los que no existen alumnos especiales ni regulares.

Al respecto, López (2014, p. 100) indica que la formación docente puede entenderse como un proceso de una sola vía, con inicio y fin, que culmina cuando se entrega el título universitario. Sin embargo, es importante entender que se trata de un proceso en donde el grado de continuidad lo determina la persona que aprende y que establece sus límites condicionada por el deseo y la necesidad de apren- 
der, de tal manera que se puede decir que la formación implica un aprendizaje a lo largo de la vida. Durante su formación profesional, los docentes reciben capacitación en lo que respecta a las características y manifestaciones de las diferentes NEE a las que pudieran enfrentarse, y aunque dicha preparación no se aborda en todos los programas de licenciatura, en el ejercicio profesional inevitablemente se van a encontrar con estudiantes con NEE.

Carvajal (2009, p. 10) señala que en la práctica docente los maestros utilizan los saberes adquiridos durante su formación y vida profesional, integran conocimientos de otras áreas y generan nuevos, al enfrentarse en su trabajo con la resolución de situaciones específicas dentro de las que se cuentan las NEE. Los cambios en la forma de ejercer la docencia no son fáciles de lograr, pero se pueden conseguir, reconociendo lo más importante, que es identificar lo requieren estos estudiantes, y asumiendo el rol de transmisor de conocimientos en un entorno de aprendizaje activo, donde el estudiante es el principal actor del proceso. Por tanto, el desempeño del docente debe ir más allá del cumplimiento de un programa y debe crear las condiciones para realizar actividades de aprendizaje fruto del análisis de su práctica, cambiando o fortaleciendo los procesos de enseñanza, teniendo en cuenta que deben estar enfocados y adaptados al modelo pedagógico institucional para que contribuyan al proceso enseñanzaaprendizaje, y aportando estrategias educativas que faciliten dicho proceso.

Las acciones docentes ante las NEE pueden considerarse desde varias perspectivas: el docente se convierte en tutor de aquellos alumnos que presentan dificultades y lleva a cabo acciones prescritas por especialistas psicólogos o psicopedagogos o, por el contrario, enseña a todo el grupo por igual. También puede darse la posibilidad de que busque por sus propios medios estrategias que ayuden a estos niños, impulsado por su vocación docente y por la valoración que hace de todos como sujetos con las mismas potencialidades y derechos. Granada, Po- més y Sanhueza (2013, p. 56) afirman que, sin duda, es importante que los profesores tengan una formación inicial profesional que les permita contar con herramientas para dar respuestas educativas de calidad a la diversidad de estudiantes en el proceso de enseñanza-aprendizaje y, por otra parte, que cuenten con una formación profesional continua que permita capacitarse y actualizarse permanentemente, para responder a las distintas demandas emergentes. En este sentido, las autoras citan algunos estudios que enfatizan la relevancia de la formación de los profesores como un factor decisivo para hacer posible el proceso de inclusión educativa. Granada et al. (2013) refieren que varios autores -Alegre, 2000; Arnaiz, 2003; Jiménez, 2005; Cardona, 2006; Sánchez et al. (2008) - muestran que el 92\% de estudiantes de pedagogía manifiesta que los profesores de educación regular no tienen la formación necesaria para atender a los alumnos con NEE.

En cuanto a la capacitación de los profesores en áreas determinadas para lograr una mayor calificación en el trabajo con estudiantes con NEE, Granada et al. (p. 56) refieren el estudio de Woolfson y Bradya (2009), que señala el hecho de que los profesores se sienten poco preparados para enseñar en ambientes inclusivos. Y es que los docentes no se autocalifican como bien preparados para incluir estudiantes con discapacidad en sus aulas ni, en general, se sienten competentes o a gusto enseñando a niños con diversas NEE. A este respecto, las citadas investigadoras referencian también a Horne y Timmons (2009) quienes enfatizan la importancia de contar con oportunidades de desarrollo profesional continuo, para así responder efectivamente al creciente número de necesidades especiales que presentan los estudiantes en la sala de clases, ya que establecen que los profesores se sienten insuficientemente preparados para cubrir las necesidades de sus estudiantes con NEE.

En Colombia, la formación docente para la atención efectiva de los alumnos con NEE es insuficiente. Así lo revelan estudios como los realizados 
por Pájaro, Sarmiento y Ramírez (2017), Jaguandoy y Guapucal (2015) y Ramírez (2016). Este último autor enfatiza en que se desconoce cómo debe construirse el currículo y los planes de área para los estudiantes con NEE, de modo que las prácticas pedagógicas en las aulas se configuran a partir de las concepciones construidas por la experiencia de los maestros y maestras, los acuerdos verbales entre los mismos y la gestión del docente del aula de apoyo (p. 146). Según Gallego y González (2014, p.158), la formación del profesorado debe ser la base sobre la que se sostiene un sistema que pretende ser inclusivo y se convierte en un reto, pues dicha formación debe promover y abarcar todos los aspectos de la enseñanza. El estudio realizado por Gallego y González con docentes del departamento del Quindío deja ver que los profesores consideran prioritario que las administraciones establezcan políticas educativas coherentes que permitan regular la inclusión de alumnos con NEE, así como atender a sus necesidades formativas en este aspecto. Requieren políticas y estrategias claras de formación, tanto inicial como permanente, y que esta se brinde de forma adecuada dentro del contexto real al que se enfrentan a diario, teniendo en cuenta sus condiciones laborales, la sobrecarga de trabajo y el aumento de la ratio profesor-alumno, factores que influyen directamente en la calidad de la educación y en la disposición de los profesionales a la inclusión (2014, p. 163).

En este marco, Horne y Timmons (2009, citados en Granada et al., 2013, p. 56) plantean que, si no se ofrece un mayor apoyo administrativo, tiempo de planificación y capacitaciones en estrategias pedagógicas específicas según discapacidades, se podría ver deteriorada la percepción positiva que los profesores tienen acerca de la inclusión.

En cuanto a los recursos institucionales con los que se cuenta para la adecuada formación de los estudiantes con NEE, Granada et al. (2013, p. 55) señalan que el tiempo disponible para enfrentar la inclusión educativa está referido a la posibilidad de contar con un lapso, espacio u oportunidad para realizar distintas acciones pedagógicas propias de la tarea educativa, como planificar, coordinar y colaborar. Sostienen las autoras que algunas de las preocupaciones de los profesores se relacionan con el tiempo disponible de planificación y la exigencia de cubrir las necesidades de todos los estudiantes. En este sentido, los recursos materiales y el tiempo continúan siendo percibidos por el profesorado como una dificultad para el desarrollo de prácticas inclusivas. Mayores oportunidades para organizar el trabajo docente podrían impactar entonces en una actitud más positiva y abierta a la inclusión de estudiantes con NEE

Los recursos de apoyo se entienden como los medios a través de los cuales se intenta dar respuestas educativas de calidad a las NEE. Dentro de estos elementos se pueden distinguir los recursos humanos y los recursos materiales. En los recursos humanos se considera la participación de expertos en áreas específicas: profesionales, asistentes, compañeros y familia. Idol (2006, citado en Granada et al., 2013, p. 55) establece que el 77\% de los educadores indica que la mejor opción para educar a estudiantes con NEE es a través de la educación general, siempre y cuando se cuente con todos los asistentes necesarios para trabajar con cada alumno que necesite apoyo. Respecto de los recursos materiales, se consideran las adaptaciones curriculares y estrategias de enseñanza-aprendizaje. En este sentido, Granada et al. (2013, p. 55) indican que cuando las escuelas tienen acceso a una gran variedad de apoyos y a diversificadas estrategias de enseñanza pueden ser efectivas incluyendo a diversos estudiantes y manteniendo altos niveles de logros académicos. También señalan que los profesores creen que necesitan clases con menos estudiantes, capacitación, tiempo de planificación y profesores asistentes de apoyo para realizar las adaptaciones curriculares necesarias.

En el contexto colombiano, Ramírez (2016, p. 150) considera que, en lo concerniente a los recursos materiales para el aprendizaje, son insuficientes o 
se encuentran en mal estado, marcados por el uso parcial de las TIC y deficiencias en la planta física de los colegios, a lo que se suma dos agravantes: por un lado, la carencia de profesionales o instituciones $u$ organizaciones públicas o de otra índole que asesoren los procesos de educación inclusiva, sobre todo en las áreas rurales de la nación, y por otro, que las prácticas pedagógicas para dar respuesta a las NEE se caracterizan por el uso de metodologías y recursos tradicionales con apoyo en un currículo diseñado para estudiantes regulares, que depende del conocimiento, habilidad, autogestión y buena fe de los docentes.

CLIMA DE AULA: se relaciona directamente con la experiencia docente, mediante la cual los maestros han diseñado estrategias para mantener el orden y la disciplina. El análisis de este factor debe partir del significado que se le otorga. Según Moreno et al. (2011, p. 72), el clima de aula es un concepto globalizador, que alude al ambiente del centro, y multidimensional, determinado por distintos elementos estructurales y funcionales de la organización, influido por las características del componente humano, como variables de especial relevancia. Y por su carácter relativamente permanente en el tiempo, el clima de aula influye en el logro de distintos objetivos académicos y de desarrollo personal y en la percepción de las personas, como indicadores fundamentales de aproximación a su estudio.

Moreno et al. (2011, p. 73) indican que un clima favorable es abierto, participativo y coherente. En un clima con estas características existiría mayor posibilidad para la formación integral del educando desde el punto de vista académico, social y emocional, puesto que se darían más oportunidades para la convivencia armónica. Pero lograrla requiere de varias condiciones, entre ellas, la disciplina. En el pasado, la disciplina en las instituciones educativas se lograba a través del miedo, es decir, con el uso del castigo, que llegó a ser incluso de tipo físico. Pero con el paso del tiempo esto se dejó atrás y se vio preferible fortalecer las conductas adecuadas que castigar las inapropiadas (Márquez, Díaz y Cazzato, 2007, p. 127).

En el orden de la educación inclusiva, el Ministerio de Educación, Cultura y Deporte de España (2012, p. 7) señala que, cuando el aula se convierte en un ambiente física y emocionalmente seguro, las expectativas de los profesores respecto de sus alumnos y de estos consigo mismos aumentan. Este ambiente está frecuentemente asociado con la existencia de límites claros a las conductas de los alumnos. Las reglas de clase son necesarias para la creación de un entorno seguro para la enseñanza y el aprendizaje, y deben evitarse aquellas que no sean necesarias o reduzcan los niveles de compromiso de los estudiantes con su propio aprendizaje. Las reglas han de aceptarse y, para que se acepten, deben satisfacer una necesidad; en consecuencia, es importante reducirlas a un mínimo. Igualmente, un sistema de premios y sanciones que pone énfasis en expectativas positivas puede promover la autoestima y la autodisciplina. Afirman los investigadores del Ministerio citado (p. 8) que los docentes eficaces estimulan a los alumnos a crecer y a aprender, sin que por ello carezcan de una dirección clara ni de control, si es necesario. Estos docentes reconocen que hay que utilizar la disciplina como una fuerza positiva y no como un medio de represión.

Para alcanzar este clima de aula en el que prime una disciplina positiva en el salón de clases compartido por estudiantes regulares y con NEE, es fundamental la actitud positiva de los docentes hacia la inclusión. Las competencias socioafectivas que muestren en relación con su práctica son fundamentales al momento de enseñar, pues no solo permiten desarrollarlas en los estudiantes, sino que también ayudan a prevenir desajustes en la salud mental de los docentes y a generar entornos favorecedores de aprendizaje. Así, el desarrollo de habilidades de estimulación afectiva, junto con la expresión regulada de emociones, la empatía, la escucha activa, la toma de perspectiva y la creación de ambientes que posibiliten la solución pacífica de conflictos 
resultan primordiales para la práctica docente y favorecen las potencialidades de los alumnos con NEE (Torres et al., 2013).

En esta misma línea, Betancur y Gómez (2015, p. 28) sostienen que el docente es quien dirige y guía el clima emocional del aula y establece qué tipo de emociones son apropiadas, cómo entenderlas y cómo expresarlas. Así mismo, los parámetros y expectativas acerca del comportamiento de los estudiantes y su desempeño académico, junto con la calidad de las interacciones entre los alumnos y entre estos y el profesor son factores que dependen de su capacidad para detectar y responder a las necesidades de los estudiantes y para construir de esta forma la relación social en su salón de clases.

RELACIÓN SOCIAL: dada la interacción día a día en el aula inclusiva, los docentes son testigos de primera mano de las relaciones sociales que se establecen entre sus alumnos con NEE y sus estudiantes regulares, así como de la independencia social que los estudiantes con NEE podrán tener en un futuro. Al respecto, la Unesco (2008, p. 10) sostiene que la educación inclusiva es un medio para avanzar hacia sociedades más justas y democráticas. Una poderosa herramienta para contribuir a la cohesión social es garantizar a toda la población una educación de calidad y desarrollar escuelas que acojan estudiantes de diferentes contextos sociales, culturas y con distintas capacidades. Así mismo, dice la Unesco (p. 11) que la educación inclusiva se sustenta en una serie de concepciones y de valores respecto del tipo de sociedad que se quiere construir y del ideal de persona que se quiere desarrollar. Si se aspira a tener sociedades más inclusivas, pacíficas y respetuosas de las diferencias, es imprescindible que los estudiantes tengan la oportunidad desarrollar y vivenciar estos valores en su proceso formativo, ya sea en escuelas o adscritos a modalidades no formales.

La relación social de los estudiantes con NEE puede verse afectada por las mismas dificultades inherentes a sus necesidades. Por lo anterior, com- partir con sus pares espacios, material de trabajo y puesta en práctica de las tareas, a través de estrategias de cooperación, contribuye a un mejor desarrollo de su autoestima y de las relaciones interpersonales y a la resolución de problemas a través de actividades que favorecen procesos cognitivos, mediante las cuales se logra al mismo tiempo mejorar la disposición del niño para acceder a los aprendizajes escolares. Así lo afirman Arismendy et al. (2009, p. 67), autoras que también consideran que en las instituciones integradoras se evidencian logros en habilidades sociales, específicamente en aspectos que tienen que ver directamente con la conducta, la participación y la independencia, los cuales tienen un impacto favorable en el desempeño del niño dentro del aula y favorecen así el proceso de integración escolar.

Las relaciones sociales positivas en el aula no favorecen únicamente a los estudiantes con NEE, pues los alumnos regulares también se benefician, ya que pueden desarrollar conductas prosociales, empatía, tolerancia y respeto por la diferencia, al punto que disminuyen las conductas agresivas, todo ello de la mano de un profesor con actitud favorable a la inclusión, que se vale de su formación, experiencia y cualidades personales para desarrollar en sus alumnos habilidades sociales que trasciendan la escuela y se manifiesten en los demás contextos en los que interactúan.

Desarrollo emocional: la ventaja de la inclusión en el aula regular de estudiantes con NEE debe verse más allá de los beneficios académicos que se puedan lograr con ella, ya que también puede favorecer el desarrollo emocional, entendido como el proceso de formación de identidad, autoestima y autoconfianza. Así lo afirma Fernández (2011, p. 140), al considerar que la educación es el medio más adecuado para que los alumnos con NEE puedan construir su personalidad, desarrollar al máximo sus capacidades y conformar su propia identidad, a la vez que les ayuda a configurar la comprensión de la realidad que les rodea y el contexto social que les ha to- 
cado vivir, integrando las dimensiones cognoscitiva y afectiva.

Sabeh (2002, p. 561) afirma que el yo de los niños con NEE crece y se desarrolla del mismo modo que el de los alumnos regulares; lo que sucede es que reciben influencias que con frecuencia son muy fuertes. Desde la infancia se enfrentan en muchos casos al rechazo social y a experiencias negativas en las relaciones interpersonales que los desvalorizan y frustran. Estas circunstancias conducen a la hipótesis de que los niños con NEE son un grupo en riesgo de desarrollar un autoconcepto negativo. Por lo anterior, los profesores deben contar con la preparación suficiente para crear y utilizar los apoyos, el clima en el aula y las respuestas adecuadas, a fin de lograr que el niño con NEE se sienta valorado, ya que, si la retroalimentación que se brinda en el aula es adecuada, puede tener una visión realista de sus limitaciones sin sentirse desvalorizado (p. 569).

CREENCIAS: este factor se refiere a la interiorización que los docentes han hecho de las ventajas que la educación inclusiva tiene para los estudiantes con NEE, al orientar sus prácticas y proyectos a reducir los procesos de exclusión social y con ello equiparar sus oportunidades para favorecer su bienestar personal y social como miembros activos y partícipes del grupo (Pérez, 2011, p. 8). Los profesores deberían considerar la educación inclusiva como una educación para todos, como participación, como valor y como garantía social. Así lo afirma Echeita (2006, p. 104), quien además sostiene que la educación inclusiva es, antes que nada, un valor y una actitud personal de profundo respeto por las diferencias y de compromiso con la tarea de no hacer de ella obstáculo, sino oportunidad.

En consecuencia, según Pérez (2011, p. 22), que se dé una educación inclusiva depende de y requiere un profesorado comprometido y reflexivo en su labor, centrado en que la mejora del aprendizaje de todos los alumnos pasa por tener la convicción y la disposición para trabajar colaborativamente con sus colegas a la hora de planificar, desarrollar y evaluar periódicamente su acción docente, introduciendo en su caso las adaptaciones, los cambios necesarios en su programación de aula y métodos didácticos para buscar una respuesta educativa en la que se minimicen las barreras que pudieran existir para el aprendizaje de los niños con NEE.

En Colombia, las creencias de los docentes en cuanto a la inclusión educativa son positivas. Así lo revelan los estudios de Vélez (2013), Gallego y González (2014) y Palacio y López (2016), que en sus hallazgos dan cuenta de la percepción favorable de los educadores respecto a que los niños con NEE deben acceder a la educación en aulas regulares, evidenciando su derecho a la igualdad, a no ser discriminados y a desarrollar de la mejor manera sus potencialidades, a pesar de no contar con la formación y los recursos idóneos.

\section{Conclusiones}

Entre las funciones de un entorno educativo incluyente está potenciar las capacidades de aprendizaje de los alumnos, despertar su interés y brindar los recursos necesarios para que el proceso de enseñanza-aprendizaje tenga los mejores resultados. Cuando los docentes cuentan entre sus alumnos con estudiantes con NEE, sus actitudes hacia ellos pueden verse influenciadas por diversos factores como los revisados en este escrito. Pero es posible que no se encuentren lo suficientemente capacitados para dar respuesta a dichas necesidades. Por ello, se hace necesario el trabajo individual fincado en la reflexión acerca de su práctica pedagógica, en la búsqueda de recursos y en la interacción con sus colegas; es decir, se precisa hacer uso de su motivación intrínseca para ayudar a los estudiantes con NEE, independientemente de los recursos que la institución educativa proporcione.

Los profesores, especialmente los que trabajan en instituciones públicas, tienen múltiples responsabilidades y se ven enfrentados a la "masificación" de 
la educación, marcada por grupos muy grandes a cargo de un solo docente o, lo que es peor, por la existencia de un único profesor para varios grupos, lo que dificulta brindar una adecuada atención a los que presentan NEE. Sin embargo, en el aula, los maestros pueden contribuir a que los niños superen los obstáculos que van presentándose en su camino. Esto puede lograrse conociendo su grupo de clase, identificando los estilos de aprendizaje más favorables y adecuando su metodología y estrategias pedagógicas, así como combinando estilos de enseñanza, para que los estudiantes potencien el que tienen interiorizado y aprendan a utilizar otros; es decir, salirse de la enseñanza tradicional y hacer realmente partícipe al niño del proceso enseñanza-aprendizaje.

No puede afirmarse que la responsabilidad sea únicamente de los profesores. Las instituciones educativas también tienen un gran compromiso en que las adaptaciones curriculares sean realizadas por profesionales idóneos, que estos guíen a los docentes de aula y que los directivos-docentes gestionen ante las secretarías de Educación capacitaciones en NEE para sus profesores y la consecución de los recursos humanos y materiales, así como las adecuaciones estructurales que las instituciones requieren para una atención integral a sus estudiantes con NEE.

Estas consideraciones se equiparan con las aportadas por Beltrán et al. (2015) respecto a que Colombia ha avanzado en el proceso de inclusión de los estudiantes con NEE en la educación regular, si bien la formulación de políticas públicas en este aspecto no es suficiente, puesto que se requiere de verdaderos esfuerzos por parte del MEN para lograr que una formación docente que permita la puesta en práctica de adaptaciones curriculares específicas para cada tipo de necesidad, lo cual debe complementarse con educación al profesorado dirigida a romper con los estereotipos, paradigmas y creencias desfavorables acerca de los estudiantes con NEE, de manera tal que logren apropiarse de su papel como protagonistas del cambio. Por último, se precisa que todos los centros educativos del territorio nacional cuenten con profesionales de apoyo y que se realice evaluación y retroalimentación permanente de los procesos de inclusión, para evitar la deserción escolar de estos alumnos.

\section{Referencias}

Asamblea Nacional Constituyente. (1991). Constitución Politica de Colombia. Recuperado de http://www.secretariasenado.gov.co/senado/basedoc/constitucion_politica_1991.html

Arismendy, L., Flórez, A., Góez, A., Perlaza, C. y Pérez, M. (2009). Desarrollo de las habilidades sociales en niños con necesidades educativas especiales integrados al aula regular. Trabajo de pregrado (Licenciatura en Educación Infantil Especial). Medellín: Universidad de Antioquia. Recuperado de http://ayura.udea.edu. co:8080/jspui/bitstream/123456789/913/1/doc.pdf

Alegre, M. (2000). Diversidad humana y educación. Málaga: Aljibe.

Arnaiz, P. (2003). Educación inclusiva: Una escuela para todos. Málaga: Aljibe.

Baron, R. y Byrne, D. (2002). Psicología social. 8 ed. Madrid: Prentice-Hall.

Beltrán, Y., Martínez, Y.y Vargas, Á. (2015). El sistema educativo colombiano en el camino hacia la inclusión. Avances y retos. Educación y Educadores, 18(1), 132-140. 
Betancur, M. y Gómez, N. (2015). Clima emocional del aula: una revisión sistemática. Trabajo de pregrado en Psicología. Bogotá, Pontificia Universidad Javeriana. Recuperado de https://repository.javeriana.edu.co/ bitstream/handle/10554/19083/BetancurFrancoMarialsabel2015.pdf?sequence=1

Carvajal, M. (2009). La didáctica en la educación. Fundación Academia de Dibujo Profesional. En línea.

Casas, C. y García, N. (2016). El saber pedagógico: categoría que transforma y configura la acción docente en la FUCS, una reflexión desde la especialización en docencia universitaria. Repertorio de Medicina y Cirugía, 25(2), 132-140. doi: 10.1016/j.reper.2016.02.014.

Castillo, A. (2007). Actitudes del profesorado de conservatorios sobre la integración educativa: un análisis exploratorio. Revista Española de Orientación y Pedagogía, 18(1), 47-58. Recuperado de http://rua.ua.es/dspace/ handle/10045/9992

Congreso de la República de Colombia (1994). Ley 115 por la cual se expide la Ley General de Educación. En línea.

Duk, C. y Loren, C. (2013). Flexibilización del currículum para atender la diversidad. Revista Latinoamericana de Inclusión Educativa, 4(1), 187-210. Recuperado de https://educrea.cl/wp-content/uploads/2016/o7/DOC1Flex-curriculum.pdf?×18843

Echeita, G. (2006). Educación para la inclusión o educación sin exclusiones. Madrid: Narcea.

Fernández, C. (2001). La inteligencia emocional como estrategia educativa inclusiva. Innovación Educativa, 21,133-150. Recuperado de http://www.usc.es/revistas/index.php/ie/article/view/29

Gallego, M. y González, F. (2014). Formación y perspectivas del profesorado frente a la inclusión de alumnos con necesidades educativas especiales en el Quindío. Revista de Investigaciones UCM, 14(23), 154-165.

Gavia, P. (2013). Creencias de los profesores sobre los problemas de conducta en alumnos con discapacidad intelectual. Ra Ximhai, 9(4), 245-258. Recuperado de http://www.redaly c.org/articulo.oa?id=46129004023

Granada, M., Pomés, M. y Sanhueza, S. (2013). Actitud de los profesores hacia la inclusión educativa. Papeles de Trabajo, 25, 51-59. Recuperado de http://www.scielo.org.ar/scielo.php?script=sci_arttext\&pid $=\mathrm{S} 1852-45082013000100003$

Horne, P. E. y Timmons, V. (2009). Making it work: teachers' perspectives on inclusion. International Journal of Inclusive Education, 13(3), 273-286. doi: 10.1080/13603110701433964

Idol, L. (2006). Toward Inclusion of Special Education Students in General Education. A Program Evaluation of Eight Schools. Remedial and Special Education, 27, 77-94. doi: 10.1177/07419325060270020601

Jaguandoy, M. y Guapucal, M. (2015). La formación docente como estrategia pedagógica para fortalecer la atención a estudiantes con necesidades educativas diversas en la Institución Educativa Luis Irizar Salazar del 
municipio de Barbacoas - Nariño. Trabajo final de máster en Educación desde la Diversidad. Universidad de Manizales. En línea. Recuperado de http://ridum.umanizales.edu.co:8080/xmlui/bitstream/handle/6789/2237/Guapucal_Cuasanchir_Maria_2015.pdf?sequence=1

Kaplan, C. (2004). La inteligencia escolarizada. Buenos Aires: Miño y Dávila.

López, M. (2014). La formación de los profesores y las dificultades de aprendizaje. Revista Nacional e Internacional de Educación Inclusiva, 7(2), 98-112. Recuperado de https://dialnet.unirioja.es/descarga/articulo/4773184.pdf

Márquez, J., Díaz, J. y Cazzato, S. (2007). La disciplina escolar: aportes de las teorías psicológicas. Revista de Artes y Humanidades UNICA, 8(18), 126-148. Recuperado de http://www.redaly c.org/pdf/1701/170118447007.pdf

Medina, I. (2015). Saberes de los docentes de dos instituciones educativas públicas del departamento de Boyacá ante las dificultades de aprendizaje que afectan el desempeño académico de sus estudiantes. Trabajo final de máster en Educación. Universidad Nacional de Colombia. En línea. Recuperado de http://www.bdigital.unal.edu.co/52829/1/1057578249.2016.pdf

MEN - Ministerio de Educación Nacional (2015). Adaptaciones curriculares - flexibilidad curricular. En línea. Recuperado de http://aprende.colombiaaprende.edu.co/sites/default/files/naspublic/cerrandobrechas/ Men\%C3\%BA\%2ohorizontal\%2oinferior/Adaptaciones\%20-\%2OFlexibilidad\%2ocurricular.pdf [Fecha de consulta: 17 de junio de 2018].

MEN - Ministerio de Educación Nacional (2009). Decreto 366 por medio del cual se reglamenta la organización del servicio de apoyo pedagógico para la atención de los estudiantes con discapacidad y con capacidades o con talentos excepcionales en el marco de la educación inclusiva. En línea. Recuperado de http://www. mineducacion.gov.co/1621/articles-182816_archivo_pdf_decreto_366_febrero_9_2009.pdf [Fecha de consulta: 9 de noviembre de 2017].

MEN - Ministerio de Educación Nacional (2007). Educación para todos. Al Tablero (43): 1. En línea. Recuperado de http://www.mineducacion.gov.co/1621/article-141881.html [Fecha de consulta: og de noviembre de 2017].

MEN - Ministerio de Educación Nacional (1996). Decreto 2082 por medio del cual se reglamenta la atención educativa para personas con limitaciones o con capacidades o talentos excepcionales. En línea. Recuperado de https://www.mineducacion.gov.co/1759/articles-103323_archivo_pdf.pdf [Fecha de consulta: o9 de noviembre de 2017].

Ministerio de Educación, Cultura y Deporte de España (2012). Educación inclusiva. Iguales en la diversidad. En línea. Recuperado de http://www.ite.educacion.es/formacion/materiales/126/cd/ [Fecha de consulta: 17 de enero de 2018].

Moreno, C., Díaz, A., Cuevas, C., Nova, C. y Bravo, I. (2011). Clima social escolar en el aula y vínculo profesor-alumno: alcances, herramientas de evaluación, y programas de intervención. Revista de Psicología Iztacala, 14(3), 70-84. Recuperado de http://www.revistas.unam.mx/index.php/repi/article/view/27647 
Mula, A. et al. (2002). Incidencias de las actitudes y expectativas de alumnos y profesores sobre el desarrollo del programa de integración. Universidad de Alicante.

Latorre,L.(2013). La educación inclusiva en docentes enformación:suevaluación a partirdelateoría defacetas.Folios, 37, 95-113. Recuperado de http://www.scielo.org.co/scielo.php?pid=S0123-48702013000100007\&script=sci_ abstract\&tlng=es

Pájaro, C., Sarmiento, L.y Ramírez, E. (2017). Formación docente frente a la educación de niños y niñas con necesidades educativas especiales del grado primero de la Institución Educativa Ciudad de Tunja - Cartagena de Indias. Trabajo de Licenciatura en Pedagogía Infantil. Universidad de Cartagena. En línea. Recuperado de http://repositorio.unicartagena.edu.co:8080/jspui/bitstream/11227/5154/1/PROYECTO\%2OCON\%2OAVAL\%20\%2O FORMACI\%C3\%93N\%2ODOCENTE.pdf

Palacio, N. y López, S. (2016). Normatividad de inclusión educativa y actitud docente: ¿una relación dialógica? Trabajo fin de máster en Educación desde la Diversidad. Universidad de Manizales. En línea. Recuperado de http://ridum.umanizales.edu.co:8080/xmlui/bitstream/handle/6789/2700/Palacio_Peralta_Nilva_2016.pdf?sequence=2\&isAllowed=y [Fecha de consulta: 02 de julio de 2018].

Pérez, E. (2011). Educación inclusiva y las comunidades de aprendizaje como alternativa a la escuela tradicional. Trabajo final de máster en Estudios Avanzados en Educación Primaria. Universidad Complutense de Madrid. En línea. Recuperado de http://eprints.ucm.es/15853/1/LA_EDUCACI\%C3\%93N_INCLUSIVA._TFM.pdf

Pozo, J.I. (2000). Concepciones de aprendizaje y cambio educativo. Ensayos y Experiencias, 33, 4-13.

Prieto, M. (2008). Creencias de los profesores sobre evaluación y efectos incidentales. Revista de Pedagogía, 29(84), 123-144. Recuperado de http://www.redaly c.org/pdf/659/65908405.pdf

Ramírez, E. (2016). Las prácticas pedagógicas de los maestros y maestras de educación básica primaria que apuntan a la inclusión de estudiantes en situación de discapacidad en el municipio de Arboletes. Trabajo final de máster en Educación. Universidad Pontificia Bolivariana. En línea. Recuperado de https://repository. upb.edu.co/bitstream/handle/20.500.11912/2865/TESIS\%2OFINAL\%2OEULISES\%2ORAM\%C3\%8DREZ. pdf?sequence $=1$

Sabeh, E. N. (2002). El autoconcepto en niños con necesidades educativas especiales. Revista Española de Pedagogía, 6o(223), 559-572.

Torres, N., Lissi, M., Grau, V., Salinas, M., Silva. M. y Onetto, V. (2013). Inclusión educativa: componentes socio-afectivos y el rol de los docentes en su promoción. Revista Latinoamericana de Educación Inclusiva, 7(2), 159-173.

Unesco - Organización de las Naciones Unidas para la Educación, la Ciencia y la Cultura (2008). La educación inclusiva: el camino hacia el futuro. En línea. Recuperado de: http://www.ibe.unesco.org/National_Reports/ ICE_2008/brazil_NRo8_sp.pdf 
ISSN 0123-1294 | e-ISSN 2027-5358 | Educ.Educ. Vol. 21. No. 2 | Mayo-agosto de 2018 | pp. 200-218.

Universidad de La Sabana | Facultad de Educación

Unesco - Organización de las Naciones Unidas para la Educación, la Ciencia y la Cultura (2009). Directrices sobre politicas de inclusión en la educación. En línea. Recuperado de: http://unesdoc.unesco.org/ images/0017/001778/177849s.pdf

Universidad de Panamá (2016). Acuerdos de la Reunión 11-16. En línea. Recuperado de http://www.up.ac.pa/ $\mathrm{ftp} / 2010 /$ secgral/documentos/acuerdos/academicos/2016/C_Acad_N\%C2\%Bo11-16.pdf

Vega, A. (2009). Integración de alumnos con necesidades educativas especiales: ¿existe coherencia entre el discurso y las prácticas pedagógicas ejercidas por los profesores básicos? Estudios Pedagógicos, 35(2), 189-202. doi: 10.4067/S0718-07052009000200011

Vélez, L. (2013). La educación inclusiva en docentes en formación: su evaluación a partir de la teoría de facetas. Folios. Segunda Época, 37, 95-113. Recuperado de http://www.scielo.org.co/pdf/folios/n37/n37ao7.pdf

Woolfson, M. L. y Brady, K. (2009). An investigation of factors impacting on mainstream teachers' beliefs about teaching students with learning difficulties. Educational Psychology, 29, 221-238. doi: $10.1080 / 01443410802708895$ 